\title{
Bluetooth Tracking of Humans in an Indoor Environment: An Application to Shopping Mall Visits
}

\author{
Dieter Oosterlinck $^{\mathrm{a}}$, Dries F. Benoit ${ }^{\mathrm{a}}$, Philippe Baecke ${ }^{\mathrm{b}}$, Nico Van de \\ Weghe $^{\mathrm{c}}$ \\ ${ }^{a}$ Faculty of Economics and Business Administration, Ghent University, \\ Tweekerkenstraat 2, B-9000 Ghent, Belgium \\ ${ }^{b}$ Area Marketing, Vlerick Business School, Reep 1, B-9000 Ghent, Belgium \\ ${ }^{c}$ Department of Geography, Ghent University, Krijgslaan 281 (S8), B-9000 Ghent, \\ Belgium
}

\begin{abstract}
Intelligence about the spatio-temporal behaviour of individuals is valuable in many settings. Generating tracking data is a necessity for this analysis and requires an appropriate methodology. In this study, the applicability of Bluetooth tracking in an indoor setting is investigated. A wide variety of applications can benefit from indoor Bluetooth tracking. This paper examines the value of the method in a marketing application. A Belgian shopping mall served as a real-life test setting for the methodology. A total of 56 Bluetooth scanners registered 18.943 unique MAC addresses during a 19-day period. The results indicate that Bluetooth tracking is a sound approach for capturing tracking data, which can be used to map and analyse the spatio-temporal behaviour of individuals. The methodology also provides a more efficient and more accurate way of obtaining a variety of relevant metrics in the field of consumer behaviour research. Bluetooth tracking can be implemented as a new and cost effective practice for marketing research, that provides fast and accurate results and insights. We conclude that Bluetooth tracking is a viable approach, but that certain technological and practical aspects need to be considered when applying Bluetooth tracking in new cases.
\end{abstract}

Keywords: Bluetooth Tracking, Marketing, Indoor, Experiment,

Email addresses: dieter.oosterlinck@ugent.be (Dieter Oosterlinck), dries.benoit@ugent.be (Dries F. Benoit), philippe.baecke@vlerick.com (Philippe Baecke), nico.vandeweghe@ugent. be (Nico Van de Weghe) 
Spatio-Temporal Analysis

\section{Introduction}

Collecting data to develop insights into the spatio-temporal behaviour of individuals can be of interest for many domains. Crowd management, safety management, operational research and consumer research in marketing provide examples of such domains (e.g. Yamin and Ades (2009)). The specific application discussed in this paper is situated in the domain of marketing research. However, the methodology itself is not limited to this domain and many insights from this specific case can be extended to other applications. We present the case of a Belgian shopping mall that wants to track customers in order to obtain insight into their behaviour. The traditional approach is periodically (e.g. every six months) hiring market research firms that survey random customers of the shopping mall about their shopping trip. Information collected this way inherently suffers from various drawbacks, such as inaccurately reported information and sample bias (Andres, 2012). Tracking methods overcome many of the disadvantages that characterize the more traditional methods. Actual paths, exact time measurement and other high quality statistics can be obtained. Still, surveys have some advantages that cannot be ignored, such as the possibility to gather more in-depth information about certain consumer preferences and characteristics (e.g. age, gender). Depending on the desired kind of information and the available budget, an adequate combination of both methods can be used.

Tracking individuals requires some form of identification. Previous research mainly used Radio Frequency Identification (RFID) technology for this purpose (Liao and Lin, 2007; Kanda et al., 2007; Hurjui et al., 2008; Takai and Yada, 2010; Fujino et al., 2014). However, unlike Bluetooth tracking, this methodology suffers from various specific drawbacks (see Section 2.2.3). This research is to our knowledge one of the first applications of Bluetooth tracking in a real-life indoor retail setting. The real-life use-case examines and demonstrates the value and possibilities of the method. The findings have implications that go beyond the specific setting of a shopping mall.

In the remainder we discuss the application of Bluetooth technology with the purpose of tracking humans in an indoor environment. In Section 2 we

review literature concerning tracking in general. We also discuss the use of Bluetooth for positioning purposes and touch upon developments in indoor 
positioning in general. Section 3 discusses the design of the study, along with some important preceding test cases that were used to optimize the methodology. The results are reported in Section 4. Section 5 summarizes the main findings, while we discuss some limitations and suggestions for further research in Section 6.

\section{Literature Review}

\subsection{Customer Tracking}

Researchers have studied the movement of people in different contexts and on various scales. Specifically for marketeers, tracking data provides valuable information. Larson et al. (2005) state that a better understanding of the shopping process could lead to important discoveries for retailing. Knowledge about in-store customer behaviour by means of path tracking is beneficial for improving Customer Relationship Management (CRM) and streamlining store operations (Celikkan et al., 2011; Vukovic et al., 2012). Already in 1966, it was clear that store layout impacts shopping behaviour (Farley and Ring, 1966). Sorensen (2003) provides an illustrative example by demonstrating that a store entrance on the right side generates an average boost in sales of two dollars per customer. This placement of the entrance favours counter-clockwise movement in the store, which apparently leads to higher sales. Knowledge about customer trajectories is a treasured input for the process of optimizing store configuration. Observing customers by means of tracking their path makes it possible to position products and stores in a way that increases sales and for example draws customer attention to previously less attracting parts of the store (Vukovic et al., 2012). Customer tracking unveils information about what sections people like, dead spots and favourite recurring spots. It can also be used to better estimate and improve many key performance indicators in the field of marketing. The fact that there is a market for these ideas is illustrated by the establishment of companies that deliver exactly these insights to their customers. Examples are Senion (Senion, 2016), Crosscan (Crosscan, 2016) and Bluenion (Bluenion, 2016). These companies mainly use Bluetooth and Wi-Fi signals. More about these technologies in the context of tracking can be found in the next section (2.2). Apple's Bluetooth based iBeacon is another player in this market (Apple, 2016a). iBeacon is frequently used for push marketing applications, where customers get notifications when they are close to such 
beacon. Nevertheless, this technology could also be used for tracking and positioning purposes. However, this requires the installation of an application by users. An important advantage of the method discussed in this research is that it does not require this.

Tracking humans is technologically challenging. Research therefore commonly makes use of proxies. These are devices or objects that can be tracked more easily and are therefore used as an approximation of the location of the user. Shopping carts are a commonly used proxy for the movement of customers (Sorensen, 2003; Larson et al., 2005; Celikkan et al., 2011). In this case, the shopping cart is equipped with an RFID-tag in order to enable localisation. The dependence on a shopping cart however limits the generalisability of the method. We therefore suggest mobile phones as a more suitable proxy, since almost every individual carries it along at all times. Also, a mobile phone is typically linked to one individual only and people typically have it in their pocket, meaning that the location of the phone very closely follows the location of the individual.

\subsection{Methods for Tracking}

Traditionally, researchers and practitioners had to rely on methods such as shadowing (Quinlan, 2008; Millonig and Gartner, 2008), collecting travel diaries (Axhausen et al., 2002) and surveys. These methods can provide rich information, but are very labour intensive. Liebig and Wagoum (2012) state that surveys are the most common method for gathering customer information, but that the high cost and the low representativeness due to the non-random sampling process strongly deteriorates its practical value. Another method that is used to track customers is the use of light curtains. A light curtain consists of two poles and is usually found at the entrance of a store. When a customer passes through the light curtain, the invisible light beam is interrupted and the visit of a customer is registered. This method is very limited, since it cannot uniquely identify customers and is therefore basically limited to visitor counts. It is impossible to construct paths, nor is it possible to calculate duration of stay or to obtain information about return behaviour. Video tracking is another approach for gathering information about customer behaviour. Saxena et al. (2008) argue that the use of video data to track individual movements forms a challenging task. The need for manually filtering out the information renders this method too labour intensive (Versichele, 2014), whereas computer vision-based alternatives need to make use of advanced image processing algorithms that may not always be 
a straightforward option (Celikkan et al., 2011). The most valuable methods for tracking are based on wireless signals. In the remainder of this section we discuss GPS, RFID, Bluetooth and Wi-Fi

\subsection{1. $G P S$}

Being explicitly designed for positioning purposes, GPS possesses interesting characteristics such as high accuracy. Unfortunately, there is no GPS coverage in an indoor environment. To overcome this issue, GPS repeaters have been developed. An outdoor GPS antenna, generally installed on the roof of a building, is connected to an indoor antenna which re-transmits the signal. Nonetheless, this solution is not sufficient in the case of tracking customers. The re-transmitted signal is limited to the location of the outdoor antenna and this would imply installing multiple, expensive repeaters per location of interest. Furthermore, this method is not applicable in a multilevel building, such as a shopping mall. Locata is another proposed solution to the indoor limitations of GPS. This local positioning system emits signals that are equivalent to GPS signals for the receiving device (Rizos et al., 2010). The system is however quite expensive. It is also more aimed at indoor positioning, not so much at tracking (see also 2.3 Indoor Positioning).

\subsubsection{RFID}

Several researchers used Radio Frequency Identification (RFID) as a technology for tracking humans (Liao and Lin, 2007; Kanda et al., 2007; Hurjui et al., 2008; Takai and Yada, 2010; Fujino et al., 2014). Due to its high positional accuracy and its applicability in indoor settings, RFID promises to be a valuable option for tracking customers in a marketing context. The methodology is however limited due to the fact that people do not simply carry an RFID-tag along. Therefore, researchers have equipped shopping carts with RFID-tags (Sorensen, 2003; Larson et al., 2005; Nakahara et al., 2010; Jung and Kwon, 2011; Vukovic et al., 2012). The advantage is that a high percentage of shoppers will be tracked. This approach however strongly limits the generalisability of the method to settings where shopping carts (or similar proxies, cf. 2.1) are available. In addition, the sample of tracked customers might be biased, since the methodology excludes shoppers without shopping cart. Customers may also leave their shopping carts behind at times, for example to quickly grasp something from a previous aisle without having to move the cart. Hence, the location of the shopping cart does not resemble the location of the customer at all times. Celikkan et al. (2011) 
also mention the high cost of using RFID, since both scanning and scanned devices (tags) need to be installed.

\subsubsection{Bluetooth}

Bluetooth has been used for tracking spatio-temporal behaviour in various domains and settings: to measure throughput time in airport security (Bullock et al., 2010), on a large scale open air festival (Versichele et al., 2012a), on a trade fair (Delafontaine et al., 2012), in an office setting (Abedi et al., 2014) and to study the movements of tourists in a city (Versichele et al., 2014a) amongst others. However, this study is to our knowledge the first application of Bluetooth tracking in an indoor retail setting.

The methodology of Bluetooth tracking uses the wireless Bluetooth signal that is a feature of most mobile phones. Bluetooth scanners are being installed at locations of interest. These scanners continuously scan for Bluetooth signals emitted by the mobile phones and register every detection. The method is based on the proximity principle. This means that the location of the device is approximated by the location of the scanner. Practically this means that if a device is detected by a certain scanner, this is registered in the log of that scanner. The path can then be reconstructed by combining the logs of all scanners, where the location of each scanner is added as the location of the device.

The Bluetooth methodology facilitates non-participatory tracking of customers (Versichele et al., 2012a). The non-participatory aspect means that 'participants' are unaware of being tracked. As such, they do not need to invest any kind of effort, which would be the case with a survey or with methods that require the installation of a smartphone application (e.g. iBeacon). It also means that the method will have no influence on their behaviour. Bluetooth tracking thus enables unbiased experiments and uninfluenced observations (Delafontaine et al., 2012). Celikkan et al. (2011) argue that in certain contexts it is acceptable that people carry a tag. A method that can avoid this dependency is however more valuable for marketing purposes. The non-participatory aspect does not mean that people cannot be individually tracked. Individual identification is made possible by registration of the unique Media Access Control (MAC) address of the device (Delafontaine et al., 2012).

Bluetooth tracking is especially useful for studying movements in 'uncontrollable' settings. We define uncontrollable settings as settings in which individuals of interest can freely move in space, without any obvious means 
of identification. In a regular supermarket for example, a shopping cart can be used as a proxy. In the setting of a festival, tags can be included in wristbands. In a shopping mall however, there is no suited proxy and the setting can be defined as uncontrollable. Many public places, such as train stations, libraries and museums can be defined as uncontrollable. Tracking individuals becomes hard in these settings and Bluetooth tracking provides an excellent methodology to keep track of the spatio-temporal behaviour of these actors. Versichele et al. (2012a) state that the methodology enables studying visitor flows at mass events. Bluetooth tracking is thus able to handle large amounts of observations. When compared to other tracking methodologies, Bluetooth can be characterized as cost effective (Abedi et al., 2014). The implementation is low cost and easy (Delafontaine et al., 2012), we nonetheless argue that the implementation should be executed with great care.

An aspect to keep in mind is that interference can affect the detection range of the Bluetooth scanners. Physical objects, radio-frequency, electronic devices and environmental factors cause different levels of interference (Harwood, 2009). Metal constructions have the strongest impact on the signal, as Agostaro et al. (2004) demonstrate. Also, Bluetooth technology was not developed with tracking or localisation purposes in mind; it is therefore not surprising that it does not have the same positional accuracy as other technologies such as outdoor GPS (Delafontaine et al., 2012). There are however ample situations in which room-level accuracy is satisfactory. Combined with the low cost, this technology will often be an interesting alternative to the genuine location-aware technologies.

Bluetooth has three operational states; off, on-invisible, on-visible (Abedi et al., 2015). It is the user of the mobile phone who chooses one of these states. The default setting varies amongst brands and devices. Only the latter of the three states is detectable for the Bluetooth scanners. This makes that only a certain ratio of the population will actually be tracked. Researchers found different detection ratios, 7\% (ONeill et al., 2006; Hagemann and Weinzerl, 2008), 11\% (Versichele et al., 2012a), 13\% (Versichele et al., 2012b) and 8\% (Versichele et al., 2014b). The fact that users are able to switch to an invisible state is negative for the sample size in a nonparticipatory approach. However, this also means that in settings where participation is allowed or even required, people can easily be asked to switch to the on-visible state. 


\subsection{4. $W i-F i$}

Mazuelas et al. (2009), Bonne et al. (2013), Danalet et al. (2014) and Abedi et al. (2015) demonstrated the use of Wi-Fi for tracking purposes in various settings. Tracking by means of Wi-Fi signals is very similar to the Bluetooth approach. Both methods use the unique MAC address to identify individuals and most (dis)advantages are also applicable in the case of Wi-Fi. In terms of data, the same information can be captured. When compared to Bluetooth, Wi-Fi tracking might have a lower accuracy, since Bluetooth is more specifically designed for short range wireless connections. Furthermore, people might be less likely to use Wi-Fi when they are away from home, since they can use mobile broadband more conveniently, whereas there are no immediate alternatives for Bluetooth, definitely not in the same device. This could lead to the conclusion that Bluetooth is the more mobile technology of both and therefore lends itself better for tracking purposes. However, Abedi et al. (2015) report a higher detection ratio for $\mathrm{Wi}-\mathrm{Fi}$, compared to Bluetooth. The conclusion is that both technologies are very analogous with regard to the application of tracking. Our assessment of the methodology with Bluetooth scanners therefore also provides insights for the use of Wi-Fi.

\subsection{Indoor Positioning}

Researchers have suggested Bluetooth not only as a tracking, but also as a positioning technology (Anastasi et al., 2003; Feldmann et al., 2003; Hallberg et al., 2003; Madhavapeddy and Tse, 2005; Rodriguez et al., 2005). For both purposes, the same Bluetooth signal and similar devices can be used. Tracking and positioning however differ to the extent that the initiator is different. Whereas for tracking the initiator is an external party (e.g. shopping mall management) that wants to gather information about the location of devices (e.g. as a proxy for a customer), for positioning, the initiator is the smartphone owner. The smartphone owner wants to know his or her own position, usually in order to reach a certain destination. In positioning cases people need to install an application, which then calculates their position with respect to static base stations.

The location of the Bluetooth scanner can be used as an approximation of the location. This approach is known as the proximity principle (Bensky, 2007) and also often referred to as the node-based approach (Nolte and Lynch, 2007). A more complex approach uses Received Signal Strength Indication (RSSI) values. These values are negatively correlated with distance 
(see also Figure 6). Therefore, it is theoretically possible to calculate a reasonably accurate location in the presence of multiple base stations. Multiple stations are required in order to enable some form of multilateration, triangulation or fingerprinting (Agostaro et al., 2004). The basic principle of these techniques is that if one knows the distance (approximated by the RSSI value in our case) to multiple points, it is possible to calculate a more refined estimate for the actual position. Several authors argue that RSSI is not a straightforward means to obtain accurate positioning (Soh et al., 2007; Versichele et al., 2010). The main problem is that the $2.4 \mathrm{GHz}$ frequency that Bluetooth uses, behaves unstable (Helen et al., 2001). Helen et al. (2001) therefore argue that the use of RSSI values as such is insufficient. Applying an Extended Kalman Filter (EKF) is suggested to improve accuracy. EKF is the nonlinear interpretation of an ordinary Kalman Filter (KF). Kalman Filtering uses Bayesian inference to estimate a result from multiple measurements, in order to be more precise than a single measurement. It is a widely used concept in navigation software (Chen et al., 2014). The search for more than room-level accuracy might however be irrelevant in many applications (Cheung et al., 2006; Hay and Harle, 2009; Fernandes, 2011). In this research for example, room-level accuracy was sufficient and therefore the proximity principle could be used. The low cost characteristic of Bluetooth and the simplicity of the proximity principle make it a compelling option in situations where the trade-off between cost and accuracy balances in favour of cost.

$\mathrm{Gu}$ et al. (2009) argue that few commercial solutions for indoor positioning are available and that they are costly and complex to install. The same companies that are involved in indoor positioning often also provide tracking applications. As mentioned before, the technological part of both applications is usually quite similar. A distinction can be made between initiatives that focus mainly on the software side versus the ones that focus mainly on the hardware side. The first category comprises initiatives that participate in indoor mapping. Examples are Google Maps Indoor (Google, 2016), Apple Indoor Maps (Apple, 2016b) and Open Street Maps (OSM, 2016). The more hardware focussed initiatives either use existing technologies, develop new ones or make a combination. Whereas GPS is the standard technology for outdoor positioning, there is no such standard at this point in time for indoor positioning. The question remains if and when there will be such technology. The answer might lie in a combination of different technologies, where Bluetooth can be one of these. Given such a standard, another 


\begin{tabular}{llll} 
Class & Radius $(\mathrm{m})$ & Max. permitted power $(\mathrm{mW})$ & Use \\
\hline Class-1 & 100 & 100 & Mainly industrial use \\
Class-2 & 10 & 2.5 & Mobile phones, carkits,... \\
Class-3 & 1 & 1 & Very short range devices \\
& & & $($ e.g. keyboard, mouse,$\ldots)$
\end{tabular}

Table 1: Bluetooth Classes

question is whether the approach will be infrastructure-free or infrastructurebased. Leveraging existing Wi-Fi networks or using geo-magnetic and sound signals by means of fingerprinting is infrastructure-free. Google Maps Floor Plan Marker is a good example here. Fingerprinting maps the space in terms of signal strength. Based on these registered values, it is possible to make a reasonable estimation of the location of a certain device. Installing beacons that use RFID, Bluetooth or other technologies are infrastructurebased approaches. The Microsoft Indoor Localization Competition provides a yearly test field for both infrastructure-free and infrastructure-based solutions to indoor positioning (Lymberopoulos et al., 2015). In the 2014 edition, a Bluetooth based solution ranked 10th out of 22 in a ranking that is mainly determined by accuracy and to a smaller extent by cost and simplicity.

\section{Experimental Design}

\subsection{Equipment}

The scanners in this research make use of Bluetooth signals. Bluetooth is situated in the ISM-band (Industrial, Scientific and Medical) at a frequency of $2.4-2.485 \mathrm{GHz}$ (Bhaskar et al., 2013). The ISM-band is shared by other technologies, such as Wi-Fi and NFC. Frequency hopping is used in order to bypass interference in this ISM-band (Bensky, 2007). Bluetooth devices are categorised into three classes, depending on their energy level (see Table 1). Most portable devices are Class-2, with a detection range of approximately 10 metres and an energy level of $2.5 \mathrm{~mW}$.

The Bluetooth scanners consist of three parts: a calculating unit, a storage unit and a Bluetooth sensor. The first part is detailed as follows: an Alix 2D2 embedded board equipped with a $500 \mathrm{MHz}$ AMD Geode LX800 processor and 256 MB DDR RAM. The storage unit comprises a 1 GB Industrial CompactFlash (CF). Three different Bluetooth sensors are used in this research: D-Link DBT-122 (Class-2), Sena UD100 (Class-1) and Trust 15542 (Class-2). A USB cable connects the sensor to the motherboard. The 


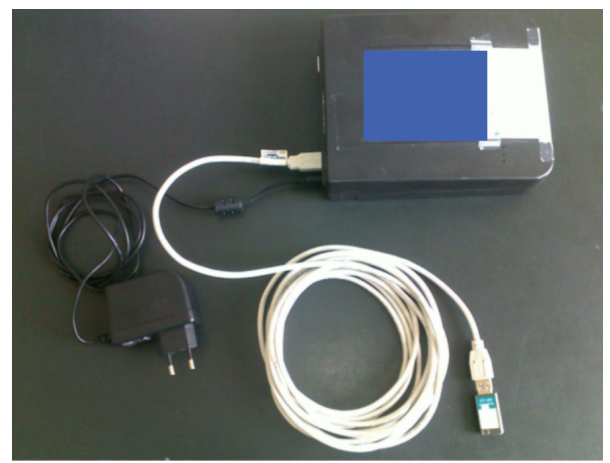

Figure 1: Bluetooth Scanner

entire device is enclosed within a protective plastic cover. A $18 \mathrm{~V}$ power cord adapter provides the scanner with electricity. (See Figure 1).

Our scanning software is installed onto the CF card. The scanners are programmed in a way that they constantly scan for 'visible' devices within their range. The scanner subsequently writes the retrieved MAC address to a log file on the CF card. Along with the MAC address, a time stamp, state and device class are registered. The MAC address is a 48-bit long number that uniquely defines the device. The first six hexadecimal digits identify the manufacturer of the tracked device (also called the Organizationally Unique Identifier). The last six hexadecimal digits represent the series number (Bhaskar et al., 2013). In order to avoid privacy issues, the 'friendly name' of the device is discarded (cf. 5. Discussion). In our experiment, the registered state can take on three distinct values: in, out or pass. 'In' represents a first detection of a certain MAC address by scanner, 'out' represents the last detection. The 'pass' state is used for solitary detections. This way of encoding the data strongly reduces the amount of data, with zero loss of valuable information. The RSSI values are stored in a separate $\log$ file.

The log files can easily be transferred through connecting a USB stick to the scanner. The data is manually transferred every two days in order to quickly identify problems. In a more continuous setting, data could also be transferred by integrating the scanners in a network. This approach is used in Versichele et al. (2014a). 


\subsection{Description of Study Area}

The actual study took place in a Belgian city centre shopping mall, during a three-week period. The shopping mall houses 39 commercial premises, divided over a below surface, ground level and first floor storey. Higher floors within the building are devoted to offices and apartments. Level -3 and -2 accommodate parking facility, connected to the shopping mall by elevators and staircases. There are three main entrances and one side entrance to the shopping mall.

The 56 scanners were set up in strategic places. Scanners were placed in the commercial premises, at the entrances of the mall, in the parking facility and around the offices. The latter for example, makes it possible to distinguish non-customers, who only pass through the shopping mall to reach their offices. To investigate whether enough devices could be detected to make meaningful conclusions, a preliminary test was performed. During two full days, a scanner placed at the main entrance scanned 3,491 unique MAC addresses. An extensive part of this number represents passers-by. Nevertheless, this result indicates that a sufficient sample size can be obtained with this approach.

\subsection{Test Cases}

Previous research used similar equipment (Versichele et al., 2012a, 2014b). These experiments were executed in an outdoor environment and with a far less dense spreading of the scanners. It is therefore crucial to pre-test certain aspects of the methodology, before initiating the actual shopping mall experiment. Three test cases were developed. The main goals were to identify issues and investigate the applicability of the proximity principle. In other words, we investigate whether using a detection by scanner $x$ (located in store $x$ ) as an indication of an actual visit to store $x$ is a valid approach. The first two test cases took place in stores of the shopping mall, whereas the third case took place at the research facility.

The first test case was executed in one store of the shopping mall (Store III, see Figure 2). The D-link DBT-122 Class-2 sensor should theoretically detect devices within a range of 10 metres, as displayed in the figure by the sphere. The used test device (Nokia E71) was nonetheless detected far beyond this theoretical range. The most distant captured point (bottom right in Figure 2), is almost 30 metres separated from the scanner. The Nokia E71 was not detected in neighbouring Store II, due to the fact that a load bearing wall separates both stores. The scanner did however register the test device 


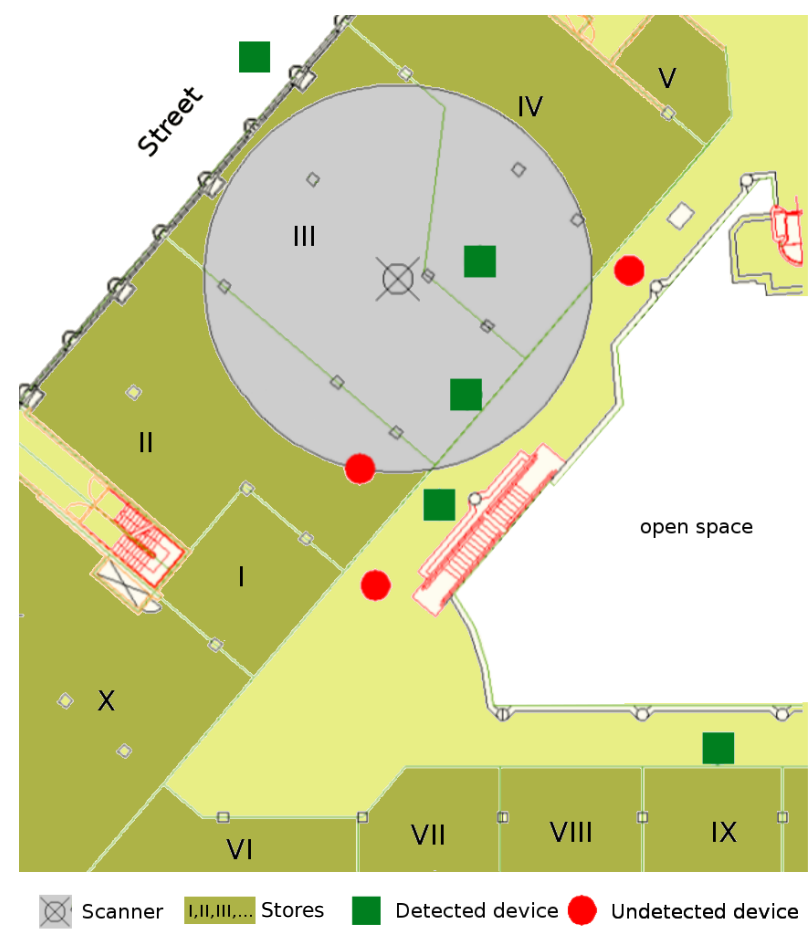

Figure 2: Test Case 1: Detection of Bluetooth Signal. The test device was detected outside the theoretical range. Squares indicate detections, whereas the circles indicate places where the device could not be detected.

in neighbouring Store IV. In this case the wall is not load bearing. These preliminary findings stress the importance of tuning the methodology when applied in an indoor environment.

Since Test Case 1 also revealed the possibility of tracking devices outside in the adjoining street, the same ground level store was selected for the second test case, along with its two neighbouring stores (see Figure 3). The choice for ground level also enables the assessment of possible detections above and below the selected stores. The Bluetooth scanners are positioned deep in the store, in order to limit the number of detections of people who just pass by the entrance. Few devices were tracked in Store II due to the fact that the scanner was placed above metal shelves. The test case revealed that the RSSI values of the registered MAC addresses fluctuate to an extent that it is not straightforward to determine whether a captured device is actually situated 


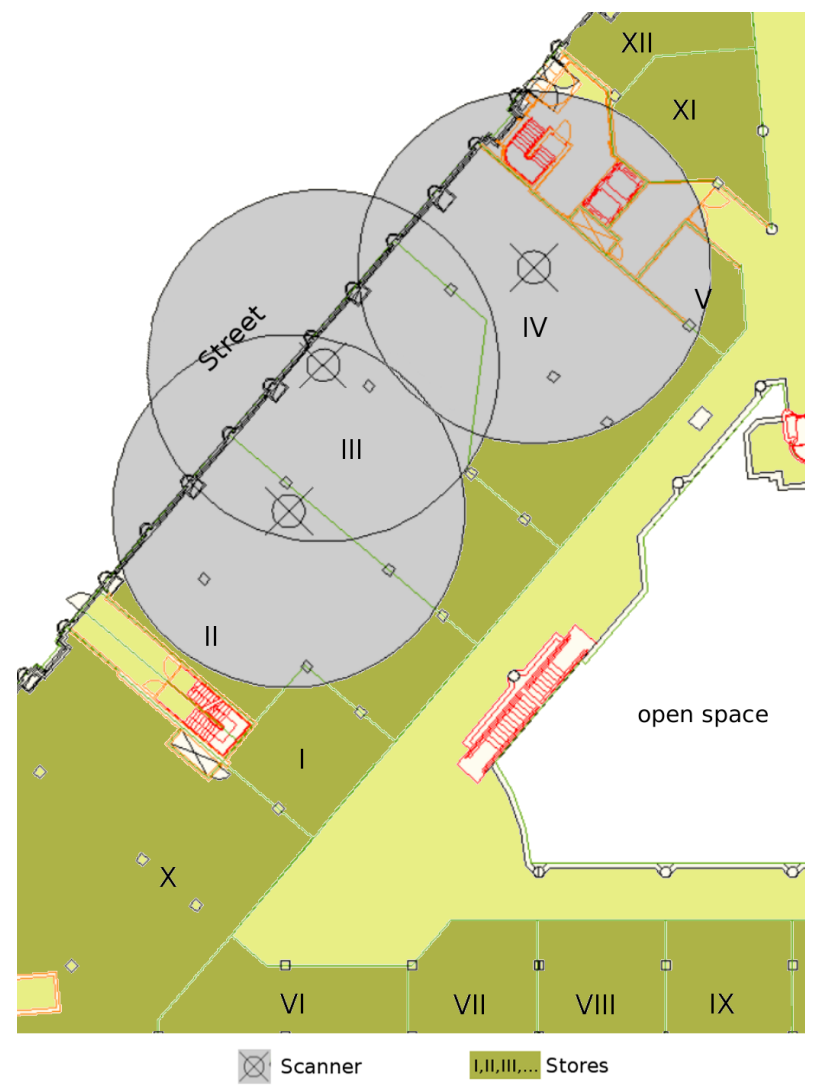

Figure 3: Test Case 2. Positioning of scanners in three neighbouring stores.

inside the store. This issue is input for Test Case 3, in which a solution is proposed. Nevertheless, one has to be aware of the fact that even after applying that solution, noise in the data is inevitable. Type I and Type II errors can occur. Type I, the more likely case, comprises devices that are registered as having visited a certain store, while in reality the person visited the neighbouring store or was only window shopping. A Type II error occurs when devices that did enter the store are not registered. This error type is however unlikely to occur.

The third test case investigated the relation between distance and RSSI. An indoor office space served as the first indoor test setting. The space was measured by means of a Pentax R-325. Forty-five markers on the ground were measured (see Figure 4). A scanner with a D-link DBT-122 sensor was 


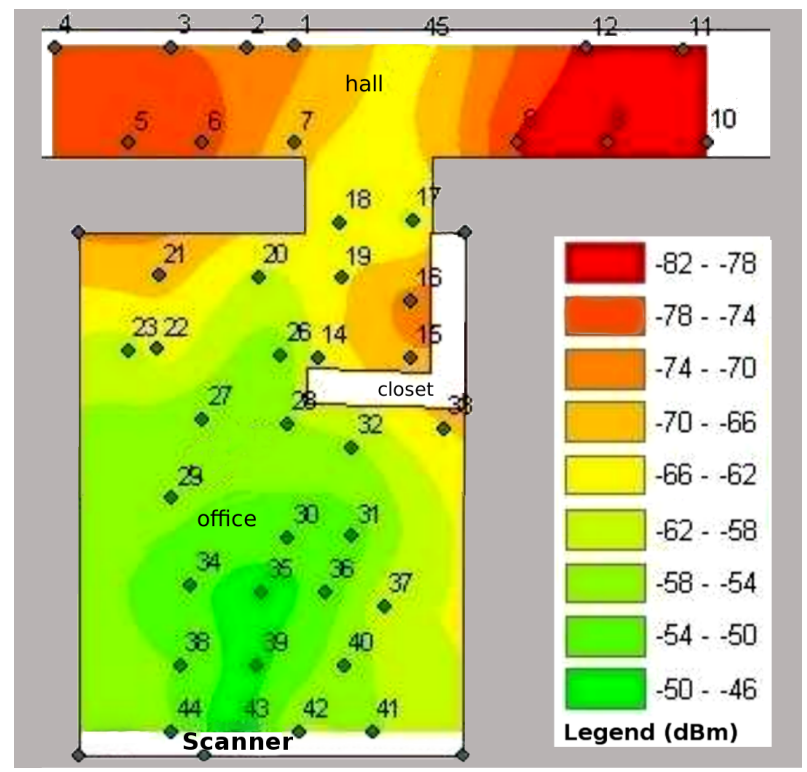

Figure 4: Test Case 3a: Interpolation of RSSI values. The figure shows a decay that is not perfectly circular, but a clear directionality is present. The figure maps an office space and a corridor, separated by a wall. The forty-five numbered points indicate the discrete points where signal strength was measured.

used to track the Nokia E71 test device. Figure 4 displays the measured values in terms of $\mathrm{dBm}$. $\mathrm{dBm}$ is an alternative to RSSI that also expresses signal strength. The only difference between both units of measurement is that RSSI is a relative value, whereas $\mathrm{dBm}$ represents absolute power levels, expressed in milliwatts. The closer $\mathrm{dBm}$ is to zero, the stronger the signal. Figure 4 is generated through interpolation of the forty-five measured values. An important finding is that the decay is not perfectly circular, but a clear directionality is present. The mirrored L represents a metal closet and shows the influence of obstacles on the signal. This aspect is crucial to keep in mind when deploying the methodology in an indoor setting, which is usually characterised by all kinds of obstacles (Feldmann et al., 2003; Zhou and Pollard, 2006). This aspect can also be used to the advantage of the researcher, by creating or making use of obstacles to define the area. A second measurement in the same room resulted in a very similar map.

A simple, but effective practice can significantly enhance the workability of the proximity principle. Wrapping the Bluetooth sensor with aluminium 


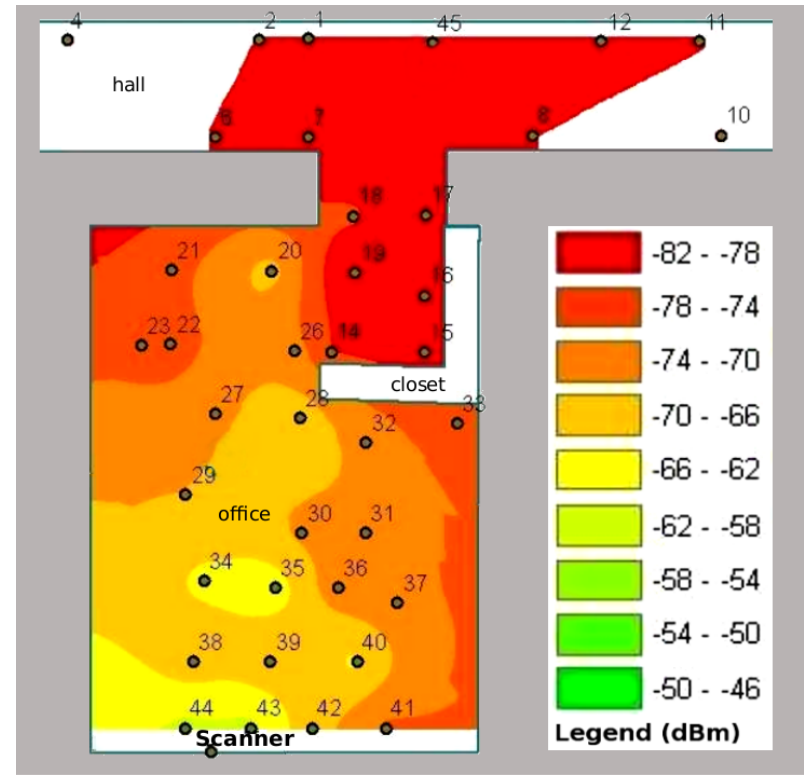

Figure 5: Test Case 3b: Interpolation of RSSI values, scanners wrapped with aluminium foil.

foil creates the effect of a Faraday cage (Cheung et al., 2006), which results in a much faster decay of the signal. This strongly reduces the chance of tracking devices outside the room of interest. With this simple trick, it is much more straightforward to define a threshold for the RSSI. The resulting values can be found in Figure 5 .

In order to test the relation between RSSI and distance without the interference of objects and materials that are typically found indoor, an outdoor evaluation was executed as a final part of the third test case. Three Bluetooth sensors were tested. The results are displayed in Figure 6. Two conclusions can be drawn from this figure. The first is that there indeed is a negative relation between distance and RSSI. The strength of this relation is emphasized by the high $R^{2}$ values. The second conclusion is that there is a difference between the different brands of Bluetooth sensors. It is not surprising that the Sena UD100 is on top, since this is a Class-1 device. However, the difference between D-link and Trust is more surprising, because those are two Class-2 devices. It is therefore important to maintain consistency in the use of a certain type of sensor throughout an experiment.

The test cases show that one needs to consider many aspects when de- 


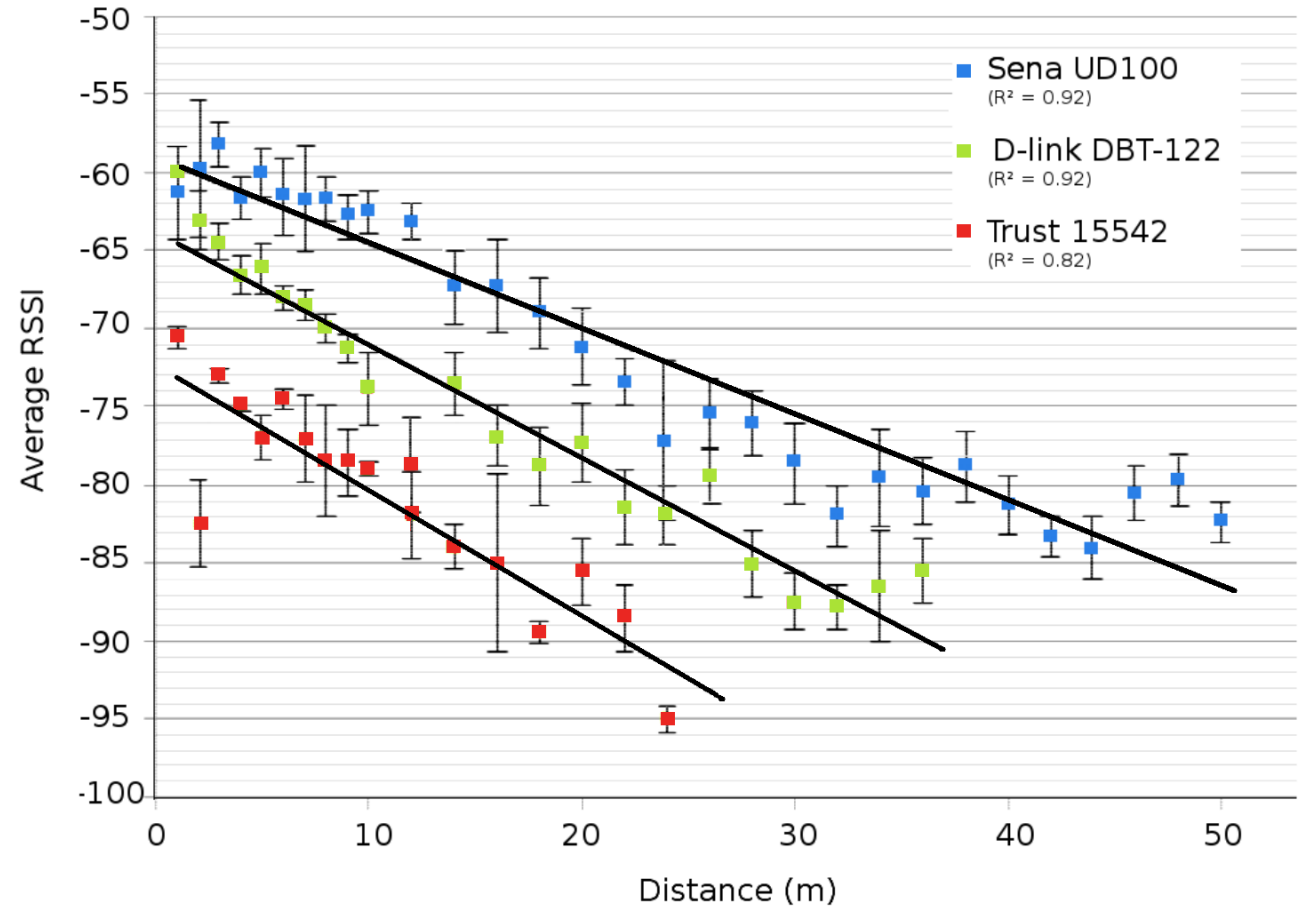

Figure 6: Test Case 3c: The Relation between RSSI and Distance. An inverse relation is clear. The test also revealed differences between brands. 
ploying Bluetooth tracking in an indoor environment. In the actual shopping mall experiment, three different types of scanners were used, optimised in function of the particular space. Aluminium foil covered multiple scanners in order to limit their reach.

\section{Results and Analysis}

\subsection{Data}

A well thought-out data filtering method can significantly improve data quality. Therefore, after installing the 56 Bluetooth scanners on site, the RSSI values at the entrance of every store were determined. These provide cut-off values for the observations such that if the measured signal strength falls below the threshold, the observation is removed. Test Case 3 provided evidence for the use of this methodology. Observations that were only tracked for a very short period can be categorised as passers-by, rather than as an actual visitor of the store. Based on that information, these observations are eliminated. Dependent on the type of store, the optimal threshold for this time interval may be different. It is therefore advisable to consider every store individually. This process reduces the number of Type I errors, but inevitably also leads to a higher number of Type II errors. However, the reduction in Type I error is much more outspoken than the increase in Type II error. In the few cases where a MAC-address was detected at the same time by two scanners, the one with the highest signal strength was selected.

As could be expected, the data comprises multiple recurring visitors. Since we are mainly interested in the paths per visit, the observations were divided into trajectories of maximal 12 hours, aligned with the opening hours of the mall. Depending on the setting and the desired analysis, the optimal threshold might be different and it requires no more effort than simply changing one parameter to enable other analyses.

We used two methods to determine the detection ratio. Method 1 concerns a manual count, based on video footage of a security camera. The one and a half hour footage resulted in a head count of 1,121 visitors. 106 distinct MAC addresses were scanned at the same location. This results in a ratio of $9.46 \%$. The second method used the counts of light curtains in three stores, during 17 full days. These counting results in a ratio of $1,721 / 17,486$, or $9.84 \%$. Calculating a weighted average, the final reported ratio is $9.81 \%$. This value lies within the expected range of values found in literature (cf. 2.2.3). 
Nineteen days of scanning customers resulted in 18.943 unique MAC addresses. When excluding the MAC addresses that were only registered in the parking or at an entrance, we get 10.719 unique devices that actually visited a store in the shopping mall. Different device types were registered. Mobile phones and smartphones accounted for $89 \%$ of the registered devices. These devices therefore possess all necessary characteristics of a good proxy. They are linked to the individual and one can therefore be confident about a one-to-one relationship between the location of a scanned MAC address and the loctaion of an individual. They are also by far the most popular category of tracked devices. The remaining $11 \%$ goes to audio/video devices (mainly headsets and carkits, accounting for 9\%), wireless phones (1\%) and computers $(1 \%)$.

\subsection{Applications}

Below, we present some applications and results. First of all, to determine which stores attract the most visitors, one could simply calculate the ratio of unique MAC addresses that were scanned in the store to the total number of unique detected MAC addresses. In our case, the analysis reveals that $75 \%$ of detected visitors visits the most popular store. This result confirms our prior expectations, since it is an anchor store. Anchor stores are used to generate traffic in a shopping mall (Pashigian and Gould, 1998). They attract large numbers of visitors, which subsequently are likely to visit the other, smaller stores as well. The second most popular store follows at $27.5 \%$, while the least popular store only attracts $0.5 \%$ of the visitors.

The uniqueness of a MAC address allows us to analyse multiple customer metrics. Customer loyalty can be approximated by how often customers return. This analysis can be run both on the individual store-level as on the level of the entire shopping mall. The analysis revealed that $50.3 \%$ of the public visited the shopping mall more than once in the observed period. Analysis of the data also reveals insights into the number of stores a customer visits. Figure 7 plots these findings. The distribution is quite similar for weekdays versus weekends, but people are slightly more likely to visit a higher number of stores during the weekends. These analyses can also be used to identify types of customers. How many people are drawn to the shopping centre because of one specific store, how many want to enjoy the full shopping mall experience and visit multiple stores, what is the role of the anchor store, etc. An analysis of the duration of store visits resulted in a median visit duration of 3.5 minutes, whereas the median duration of the total shopping 


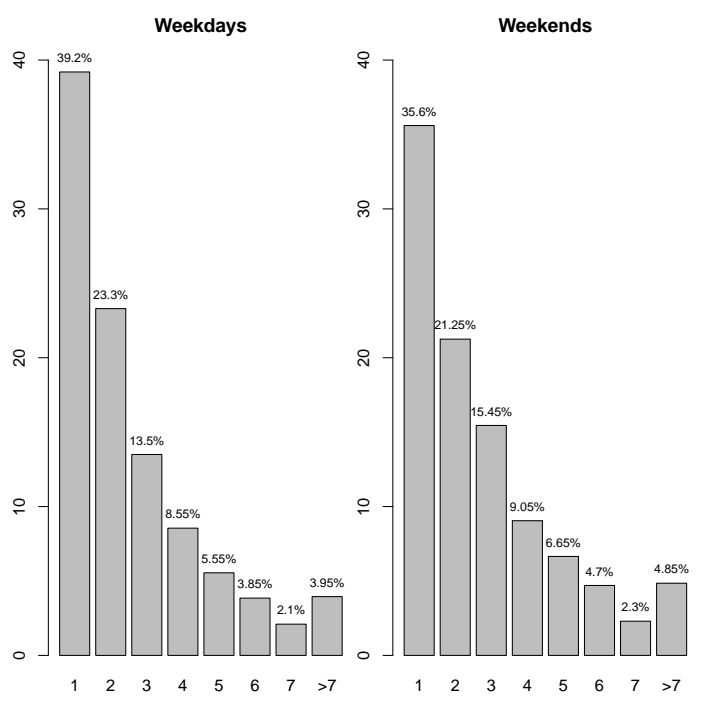

Figure 7: Number of Stores Visited (Relative Frequencies)

mall visit was 24 minutes (see Figure 8). The result of 24 minutes is in line with our expectations, since we are dealing with a rather small shopping mall of 39 stores. Very long visits can usually be attributed to employees. This provides an additional filtering method to exclude employees from the analysis, besides a more manual filtering process of explicitly scanning their MAC addresses.

Before installing the Bluetooth scanners, one has to reflect on the desired kind of information. If one of the objectives is to acquire insight in the means of transport of customers, it is crucial to place scanners in the parking facility or nearby public transport hubs. In our case, the shopping mall was mainly interested in the use of the parking facility. We calculated that during weekdays $18.6 \%$ of the customers comes by car, while this percentage grows to $31.2 \%$ during weekends. An explanation can be that during the weekend most of the customers intentionally visit the shopping centre as a planned event. These people might come from outside the city and therefore come by car. During the week visitors are probably mostly people who live or work close-by the shopping mall and therefore do not need a car to reach the shopping mall, which is located in the city centre. To confirm this hypothesis, Bluetooth scanners could be placed at multiple locations in the city and on access roads to the city. 


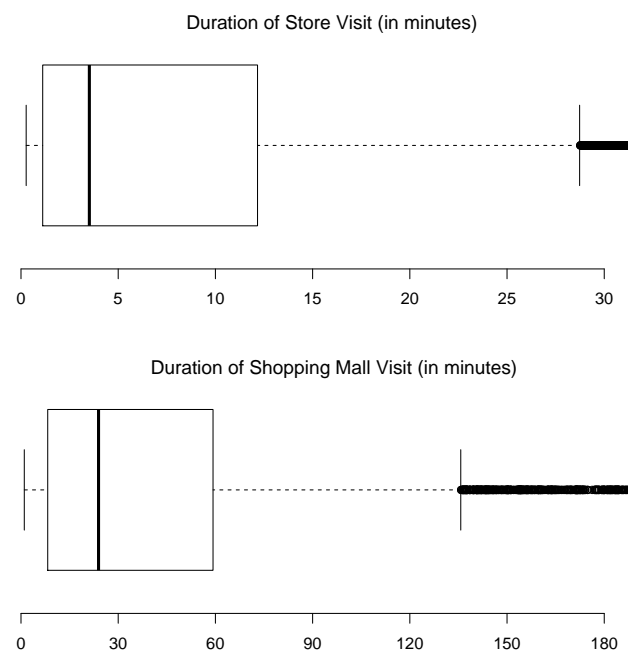

Figure 8: Time Measurement of Visits

For sequential analysis of the data an important condition arises. Since the data comes from scanners on multiple close-by locations, the timestamps have to be very precise. Therefore all internal clocks were synchronised at the beginning of the experiment.

Combining the tracking data with the map of the shopping mall makes it possible to calculate path lengths. The path length does not include the distance travelled within stores, since the spatio-temporal behaviour is recorded at store-level. The average path length amounted to 279 metres. There are several options to visualise the path data. We present two suggestions to visualise the number of customers that visit store $x$ after having visited store $y$. The first visualization is a plot of the flows on the map of the shopping mall (see Figure 9). To maintain readability of this map, the map is restricted to the most frequent flows. We also restricted this figure to one floor of the shopping mall, since mapping the flows between all shops over the three floors would result in a cluttered 3D-plot. An alternative visualization that overcomes this latter problem is a migration plot (see Figure 10). This plot displays the major flows in the entire shopping mall. It is clear that store B, the anchor store (consisting out of three departments) accounts for a large part of the flows. The first plot shows more clearly that people are likely to visit a neighbouring store as the next store. This kind of observations are 


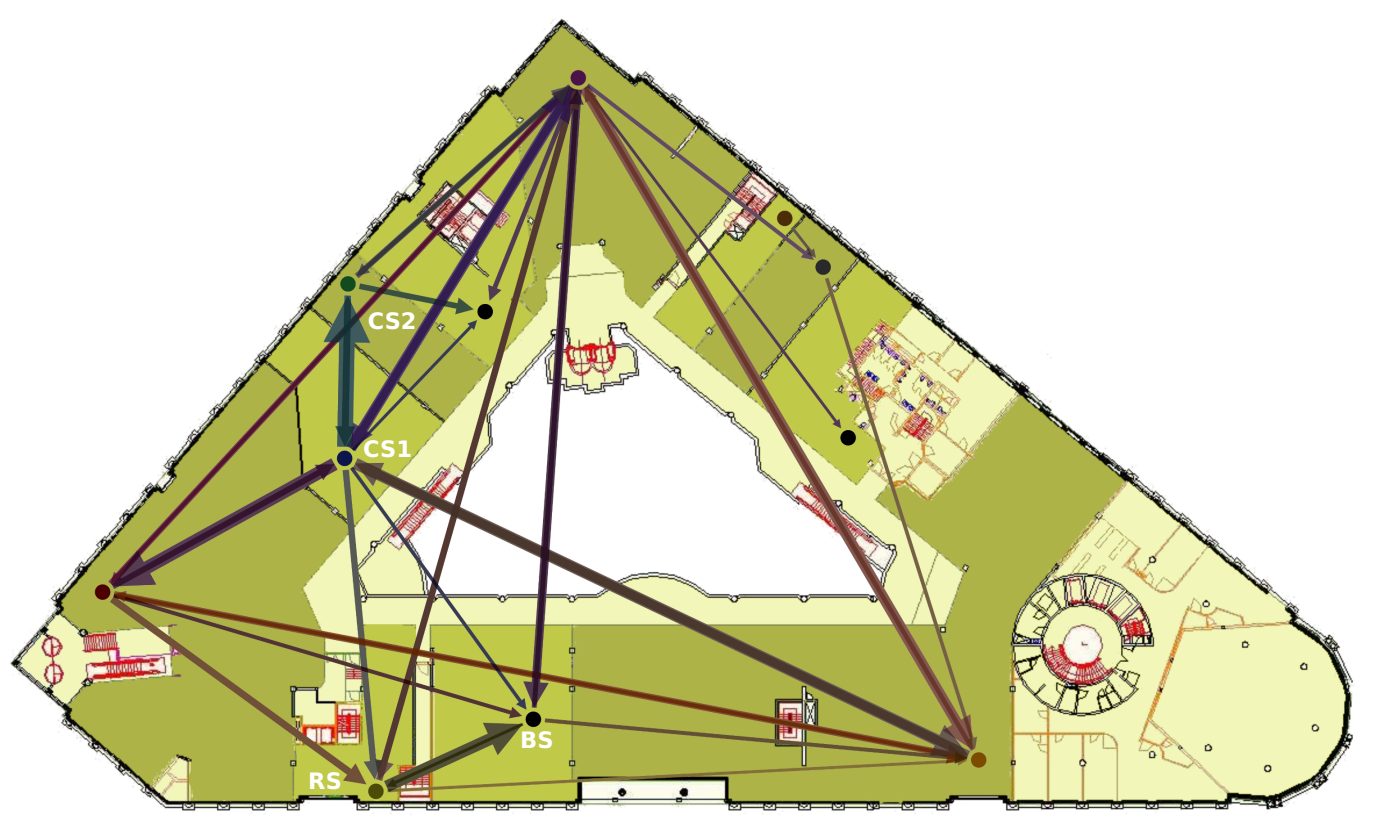

Figure 9: Major Flows on First Floor of Shopping Mall. The linewidth is relative to the size of the flow. From this figure we can observe that many people that visit the record store (RS) subsequently visit the neighbouring bookstore (BS). The same holds for the two similar neighbouring clothing stores (CS1 and CS2). 
not immediately clear from a migration plot. Hence, different visualisations can provide rich and complimentary insights.

A last application is cluster analysis on the path data. The goal of cluster analysis is to segment the vast amount of paths and visitors in order to identify groups. We clustered customers on the stores they visited, regardless of the specific order in which they visited the stores. This enables us to categorise paths such as A-B-C in the same cluster as path C-B-A. For our purpose, this latter path is more similar to A-B-C than A-B-D is for example. Depending on the desired output and insights, one needs to decide on whether or not taking the actual sequence into account. When ignoring the actual sequence, more traditional clustering methods can be used. A matrix was created in which a value of 1 was assigned to the store variable if the store was visited and a 0 in the opposite case. Based on that new dataset, we applied hierarchical clustering (Murtagh, 1985) on the entire sample of 10.719 visitors. Figure 11 shows a dendrogram for a random sample of 50 customers. The figure was restricted to this low sample number in order to maintain readability. The full analysis does nevertheless include the entire sample of 10.719 visitors. The clustering method allows the user to define the optimal number of clusters, depending on the desired level of detail. Graphically, that means drawing a horizontal line on the desired height in Figure 11. The higher the line, the less clusters will be defined. In the figure, moving the line upwards would for example imply that the two most left clusters become one larger cluster. When opting for a manageable number of five clusters, the omni-presence of the anchor store became clear again. Cluster 2, 3 and 4 each represent customers that visit almost exclusively this store. Cluster 1 contains customers that come to the shopping mall mainly for eating and multi-media stores, but not for the clothing stores. Customers who visit a variety of stores are to be found in cluster 5 .

\section{Discussion and Conclusion}

The main goal in this research was to investigate the applicability of Bluetooth tracking in an indoor environment. Many factors make it far from evident to obtain sequential path data in indoor settings. This research provides a real-life example of the application of Bluetooth tracking in an indoor shopping mall. We have shown that the method is able to analyse the spatio-temporal behaviour of individuals. The method is effective and comes 


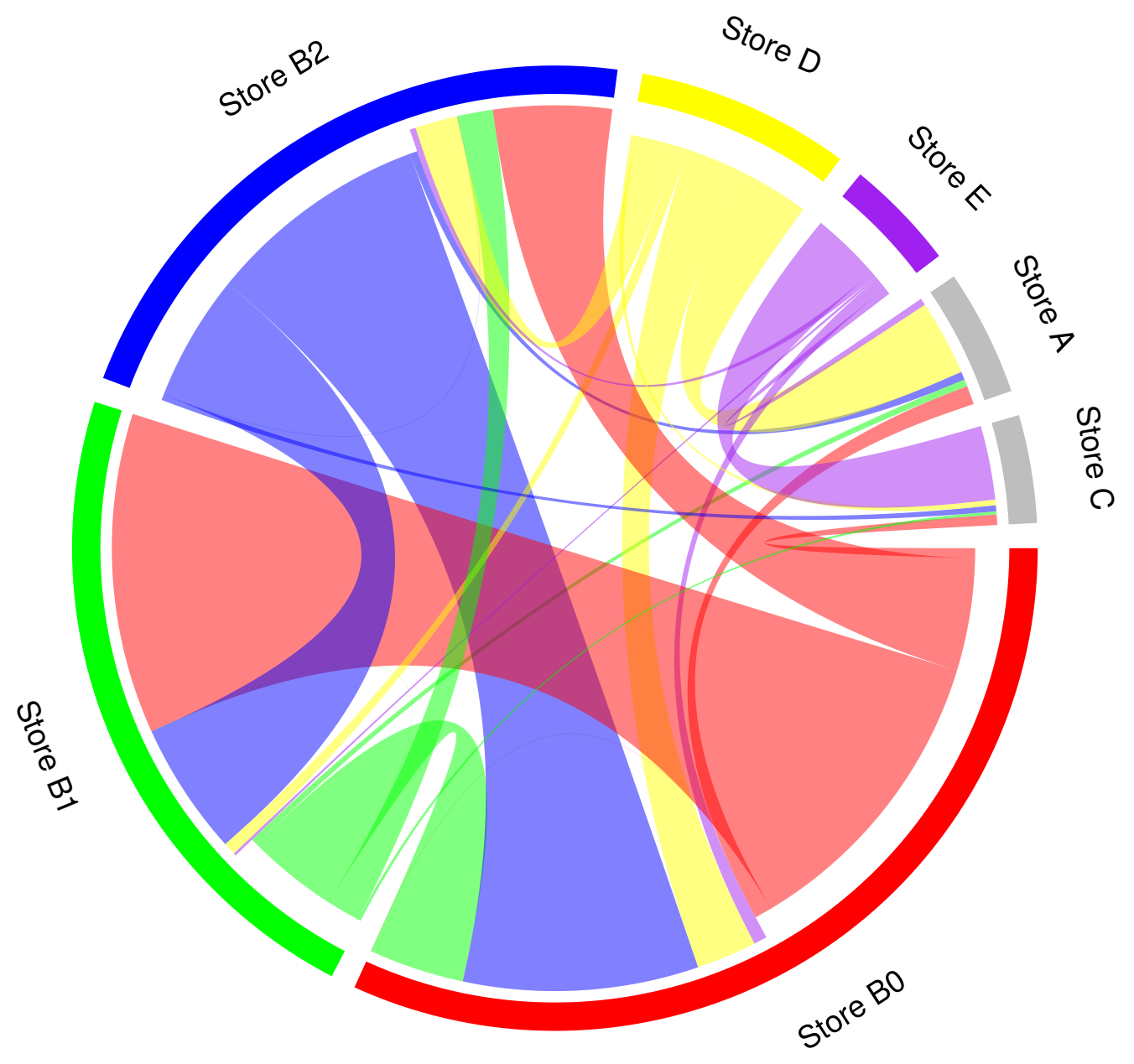

Figure 10: Migration Plot of Major Flows. The circle segments represent the different stores as origins and destinations of the flows. The width of the links represent the size of the flows. The colours/greyscale indicate the direction of the flows, links have the same colour as the origin. There is also a gap between the start of the link and the segment to indicate the origin. 


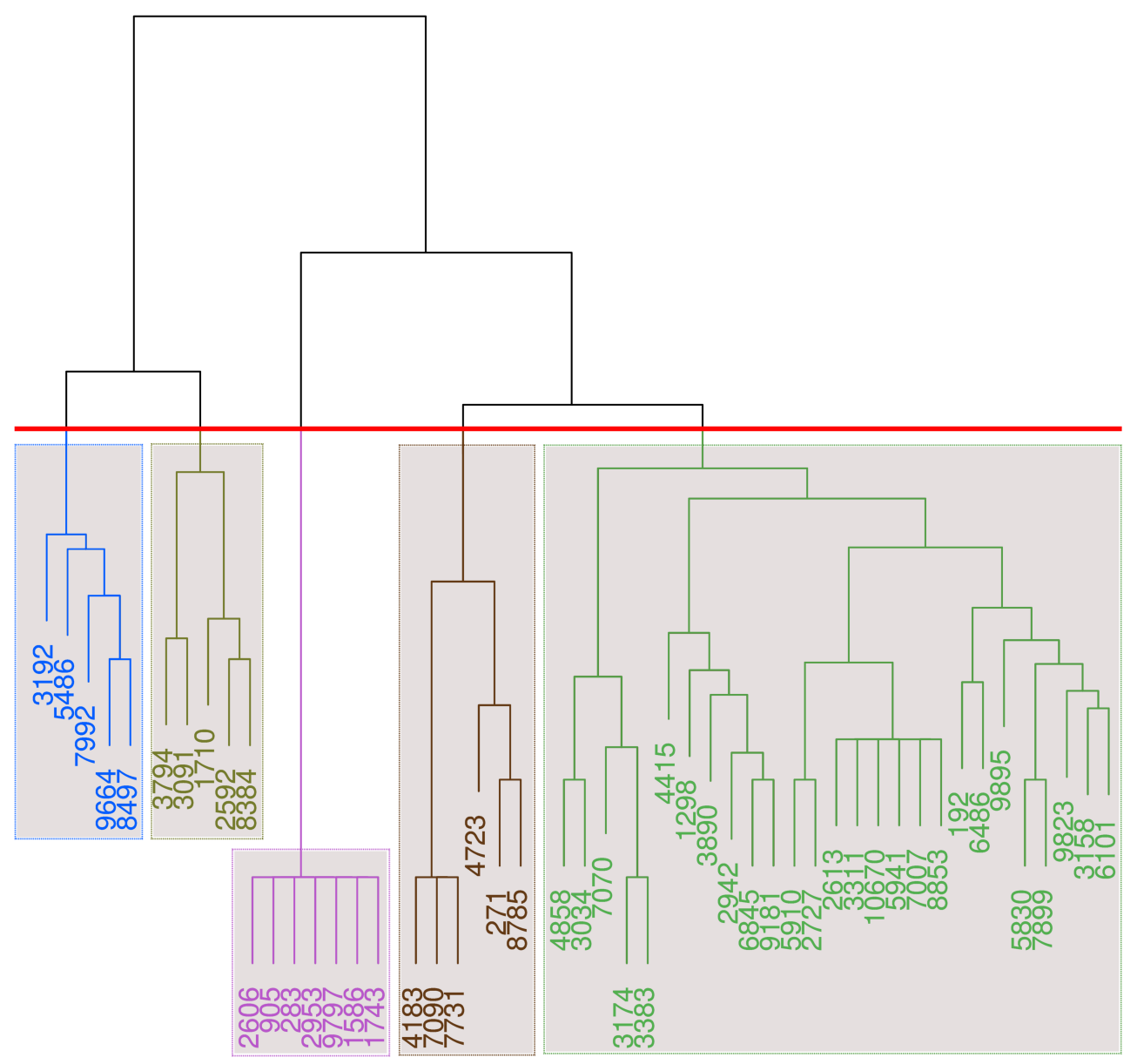

Figure 11: Cluster Dendrogram of Hierarchical Clustering on 50 Random Customers. The example shows five extracted clusters. 
at a rather low cost when compared to the competing technologies such as RFID.

Three test cases were executed prior to the actual experiment. These cases revealed that it is crucial to invest a fair amount of time and effort in the tuning of the scanning devices and their position. Different types of scanners and a wrapping of aluminium foil were used to optimize the accuracy. Additional filtering of the data forms a further step to improve data quality.

We demonstrated the relevance of knowledge and advances in the field of geography for other domains such as marketing. A shopping mall was used as research setting for this marketing application. In a broader sense, Bluetooth tracking is relevant in many settings that we defined as 'uncontrollable' (see Section 2.2.3). Also, Bluetooth is used in indoor positioning and navigation.

This research is to our knowledge not only the first to deploy Bluetooth tracking in a real-life indoor retail setting, but also the first that uses Bluetooth tracking as an alternative for gathering marketing insights. The collected data not only reveals paths as such, but can also be used to discover many other relevant statistics. Furthermore, this information is of higher quality than most of the traditional surveys. A simple example is the duration of a visit. Bluetooth tracking data provides the actual values, whereas this is not feasible for people. In addition, the non-participatory approach (cf. 2.2.3) results in natural behaviour of the tracked individuals. Of course, certain more in-depth information cannot be obtained with this method and if this is desired, a traditional survey can provide this. Furthermore, Bluetooth tracking definitely lowers the cost of information acquisition. The detection ratio of $9.81 \%$ is high enough to quickly generate a sufficiently large sample. In contrast to traditional survey methods, where the cost is proportional to the sample size, the cost stays constant in case of Bluetooth tracking. The method therefore is a sound long-term investment.

Considering the legal importance of privacy in marketing research, the non-participatory aspect should be approached with great care, since tracking human beings without their consent likely generates legal problems. A considerable advantage of Bluetooth tracking therefore lies in the use of MAC addresses. These cannot be linked to the true identity of the person (Giannotti and Pedreschi, 2008; Abedi et al., 2014). A problem with GPS tracking for example is the fact that it can easily link the person's identity through the used smartphone application (Giannotti and Pedreschi, 2008; Abedi et al., 2014). 
We can conclude that Bluetooth tracking is a sound methodology to gather information about the unbiased spatio-temporal behaviour of individuals. Furthermore, shopping mall management did consider the experiment interesting and successful. The current line of thinking is to use Bluetooth tracking next to the already existing bi-yearly customer survey.

\section{Limitations and Future Research}

The detection ratio is a significant limitation of the methodology. Devices must be in on-visible mode in order to be scanned. In our experiment, the detection ratio amounted to $9.81 \%$, which is similar to ratios found in previous research (ONeill et al., 2006; Hagemann and Weinzerl, 2008; Versichele et al., 2012a,b, 2014b). For many purposes this will lead to a sufficient sample size. Furthermore, Bluetooth tracking might introduce a sample bias. Further research is needed to investigate whether certain segments of the population are more likely to carry discoverable Bluetooth devices. The evolution of the detection ratio over time is another aspect that deserves attention. At the moment, it is not clear whether the technology usage will increase or decrease.

Another drawback of the methodology is its currently rather unautomated set-up. Covering scanners with aluminium wrapping is an inconvenient approach to limit the reach of the scanners, in order to increase accuracy. Further research should investigate whether other, more automated, procedures could attain the same effect. It might become possible to automatically program the scanners so that they only scan a specific region. Until now, a solution lies in putting a strict threshold on the RSSI values that the scanner should allow. This software approach does however not necessarily lead to better results than the hardware approach. The non-centralised data collection is another aspect that could be improved upon. As suggested by Versichele et al. (2014a), connecting the scanners via a (wireless) network might significantly improve the practical usability.

Clearly, there are downsides characterizing the Bluetooth tracking method. However, no currently existing tracking method is flawless, nor are the more traditional methods used in marketing and other research. This research shows that, depending on the context, the desired information and level of detail, Bluetooth tracking proves to be a viable approach. 


\section{References}

Abedi, N., Bhaskar, A., Chung, E., 2014. Tracking spatio-temporal movement of human in terms of space utilization using media-access-control address data. Applied Geography 51, $72-81$.

Abedi, N., Bhaskar, A., Chung, E., Miska, M., 2015. Assessment of antenna characteristic effects on pedestrian and cyclists travel-time estimation based on bluetooth and wifi MAC addresses. Transportation Research Part C: Emerging Technologies 60, $124-141$.

Agostaro, F., Collura, F., Genco, F., Sorce, S., 2004. Problems and solutions in setting up a low cost bluetooth positioning system. WSEAS Transactions on Computers 3 (4), $1102-1106$.

Anastasi, G., Bandelloni, R., Conti, M., Delmastro, F., Gregori, E., Mainetto, G., 2003. Experimenting an indoor bluetooth-based positioning service. In: Distributed Computing Systems Workshops, 2003. Proceedings. 23rd International Conference on. IEEE, pp. 480-483.

Andres, L., 2012. Designing and doing survey research. Sage.

Apple, 2016a. ibeacon for developers - apple developer.

URL https://developer apple.com/ibeacon

Apple, 2016b. Maps for developers - apple developer.

URL https://developer .apple.com/maps

Axhausen, K. W., Zimmermann, A., Schönfelder, S., Rindsfüser, G., Haupt, T., 2002. Observing the rhythms of daily life: A six-week travel diary. Transportation 29 (2), 95-124.

Bensky, A., 2007. Wireless positioning technologies and applications. Artech House.

Bhaskar, A., Kieu, L. M., Qu, M., Nantes, A., Miska, M., Chung, E., September 2013. On the use of bluetooth mac scanners for live reporting of the transport network. In: 10th International Conference of Eastern Asia Society for Transportation Studies. Taipei, Taiwan.

Bluenion, 2016. Indoor positioning and tracking system - bluenion.

URL http: //www. bluenion.com/solutions . php?id=8 
Bonne, B., Barzan, A., Quax, P., Lamotte, W., 2013. Wifipi: Involuntary tracking of visitors at mass events. In: World of Wireless, Mobile and Multimedia Networks (WoWMoM), 2013 IEEE 14th International Symposium and Workshops on a. IEEE, pp. 1-6.

Bullock, D., Haseman, R., Wasson, J., Spitler, R., 2010. Automated measurement of wait times at airport security. Transportation Research Record: Journal of the Transportation Research Board 2177, 60 - 68 .

Celikkan, U., Somun, G., Kutuk, U., Gamzeli, I., Cinar, E., Atici, I., 2011. Capturing supermarket shopper behavior using smartbasket. In: Snasel, V., Platos, J., El-Qawasmeh, E. (Eds.), Digital Information Processing and Communications. Vol. 189 of Communications in Computer and Information Science. Springer Berlin Heidelberg, pp. 44-53.

Chen, X., Wang, X., Xu, Y., 2014. Performance enhancement for a gps vector-tracking loop utilizing an adaptive iterated extended kalman filter. Sensors 14 (12), 23630-23649.

Cheung, K. C., Intille, S. S., Larson, K., 2006. An inexpensive bluetoothbased indoor positioning hack. In: Proceedings of UbiComp 2006 Extended Abstracts.

Crosscan, 2016. Real-time data solutions for retail - crosscan gmbh.

URL http://crosscan.com/en

Danalet, A., Farooq, B., Bierlaire, M., 2014. A bayesian approach to detect pedestrian destination-sequences from wifi signatures. Transportation Research Part C: Emerging Technologies 44, 146-170.

Delafontaine, M., Versichele, M., Neutens, T., de Weghe, N. V., 2012. Analysing spatiotemporal sequences in bluetooth tracking data. Applied Geography 34, $659-668$.

Farley, J., Ring, L., 1966. A stochastic model of supermarket traffic flow. Annals of Operations Research 14 (4), 555 - 567.

Feldmann, S., Kyamakya, K., Zapater, A., Lue, Z., 2003. An indoor bluetooth-based positioning system: Concept, implementation and experimental evaluation. In: International Conference on Wireless Networks. pp. 109-113. 
Fernandes, T., 2011. Indoor localization using bluetooth. In: 6th Doctoral Symposium in Informatics Engineering. pp. 480-483.

Fujino, T., Kitazawa, M., Yamada, T., Takahashi, M., Yamamoto, G., Yoshikawa, A., Terano, T., 2014. Analyzing in-store shopping paths from indirect observation with RFID-tags communication data. Journal on Innovation and Sustainability. RISUS ISSN 2179-3565 5 (1), 88-96.

Giannotti, F., Pedreschi, D., 2008. Mobility, Data Mining and Privacy: A Vision of Convergence. Springer.

Google, 2016. Indoor maps - about - google maps.

URL https://www.google.com/maps/about/partners/indoormaps

Gu, Y., Lo, A., Niemegeers, I., First 2009. A survey of indoor positioning systems for wireless personal networks. Communications Surveys Tutorials, IEEE 11 (1), 13-32.

Hagemann, W., Weinzerl, J., 2008. Automatische erfassung von umsteigern per bluetooth-technologie. Nahverkerspraxis. Springer, Heidelberg.

Hallberg, J., Nilsson, M., Synnes, K., 2003. Positioning with bluetooth. In: Telecommunications, 2003. ICT 2003. 10th International Conference on. Vol. 2. IEEE, pp. 954-958.

Harwood, M., 2009. CompTIA network+ N10-001. Pearson Education.

Hay, S., Harle, R., 2009. Bluetooth tracking without discoverability. In: Location and context awareness. Springer, pp. 120-137.

Helen, M., Latvala, J., Ikonen, H., Niittylahti, J., 2001. Using calibration in RSSI-based location tracking system. In: Proceedings of the 5th world multiconference on circuits, systems, communications and computer.

Hurjui, C., Graur, A., Turcu, C., 2008. Monitoring the shopping activities from the supermarkets based on the intelligent basket by using the RFID technology. Electronics and Electrical Engineering 83 (3), 7 - 10.

Jung, I.-C., Kwon, Y. S., 2011. Grocery customer behavior analysis using RFID-based shopping paths data. World Academy of Science, Engineering and Technology 59, 2011. 
Kanda, T., Shiomi, M., Perrin, L., Nomura, T., Ishiguro, H., Hagita, N., 2007. Analysis of people trajectories with ubiquitous sensors in a science museum. In: Robotics and Automation, 2007 IEEE International Conference on. IEEE, pp. 4846-4853.

Larson, J. S., Bradlow, E. T., Fader, P. S., 2005. An exploratory look at supermarket shopping paths. International Journal of Research in Marketing $22(4), 395-414$.

Liao, I.-E., Lin, W.-C., Sept 2007. Shopping path analysis and transaction mining based on RFID technology. In: RFID Eurasia, 2007 1st Annual. pp. $1-5$.

Liebig, T., Wagoum, A., 2012. Modelling microscopic pedestrian mobility using bluetooth. ICAART 2, $270-275$.

Lymberopoulos, D., Liu, J., Yang, X., Choudhury, R. R., Handziski, V., Sen, S., 2015. A realistic evaluation and comparison of indoor location technologies: Experiences and lessons learned. In: Proceedings of the 14th international conference on information processing in sensor networks. ACM, pp. 178-189.

Madhavapeddy, A., Tse, A., 2005. A study of bluetooth propagation using accurate indoor location mapping. In: UbiComp 2005: Ubiquitous Computing. Springer, pp. 105-122.

Mazuelas, S., Bahillo, A., Lorenzo, R. M., Fernandez, P., Lago, F., Garcia, E., Blas, J., Abril, E. J., et al., 2009. Robust indoor positioning provided by real-time rssi values in unmodified wlan networks. Selected Topics in Signal Processing, IEEE Journal of 3 (5), 821-831.

Millonig, A., Gartner, G., 2008. Shadowing-tracking-interviewing: How to explore human spatio-temporal behaviour patterns. In: BMI. pp. 1-14.

Murtagh, F., 1985. Multidimensional clustering algorithms. Compstat Lectures, Vienna: Physika Verlag, 1985.

Nakahara, T., Uno, T., Yada, K., 2010. Extracting promising sequential patterns from RFID data using the lcm sequence. In: Knowledge-Based and Intelligent Information and Engineering Systems. Springer, pp. 244253. 
Nolte, T., Lynch, N., 2007. A virtual node-based tracking algorithm for mobile networks. In: Distributed Computing Systems, 2007. ICDCS'07. 27th International Conference on. IEEE, pp. 1-1.

OSM, 2016. Indoor mapping - openstreetmap wiki.

URL http://wiki.openstreetmap.org/wiki/Indoor_Mapping

ONeill, E., Kostakos, V., Kindberg, T., Penn, A., Fraser, D. S., Jones, T., et al., 2006. Instrumenting the city: Developing methods for observing and understanding the digital cityscape. In: UbiComp 2006: Ubiquitous Computing. Springer, pp. 315-332.

Pashigian, B. P., Gould, E. D., 1998. Internalizing externalities: The pricing of space in shopping malls. The Journal of Law and Economics 41 (1), $115-142$.

Quinlan, E., 2008. Conspicuous invisibility: shadowing as a data collection strategy. Qualitative Inquiry 14, 1480 - 1499.

Rizos, C., Roberts, G., Barnes, J., Gambale, N., 2010. Experimental results of locata: A high accuracy indoor positioning system. In: Indoor Positioning and Indoor Navigation (IPIN), 2010 International Conference on. IEEE, pp. 1-7.

Rodriguez, M., Pece, J. P., Escudero, C. J., 2005. In-building location using bluetooth. In: International Workshop on Wireless Ad-hoc Networks.

Saxena, S., Brémond, F., Thonnat, M., Ma, R., 2008. Crowd behavior recognition for video surveillance. In: Advanced Concepts for Intelligent Vision Systems. Springer, pp. 970-981.

Senion, 2016. Senion indoor positioning system indoor navigation. URL https://senion.com/

Soh, W.-S., et al., 2007. A comprehensive study of bluetooth signal parameters for localization. In: Personal, Indoor and Mobile Radio Communications, 2007. PIMRC 2007. IEEE 18th International Symposium on. IEEE, pp. $1-5$.

Sorensen, H., 2003. The science of shopping. Marketing Research 15 (3), 30 $-35$. 
Takai, K., Yada, K., 2010. Relation between stay-time and purchase probability based on RFID data in a japanese supermarket. In: Knowledge-Based and Intelligent Information and Engineering Systems. Springer, pp. 254263.

Versichele, M., 2014. Sensing and making sense of crowd dynamics using bluetooth tracking: an application-oriented approach. Ph.D. thesis, Ghent University.

Versichele, M., de Groote, L., Bouuaert, M. C., Neutens, T., Moerman, I., de Weghe, N. V., 2014a. Pattern mining in tourist attraction visits through association rule learning on bluetooth tracking data: A case study of ghent, belgium. Tourism Management 44, $67-81$.

Versichele, M., Delafontaine, M., Neutens, T., Van de Weghe, N., 2010. Potential and implications of bluetooth proximity-based tracking in moving object research. In: 1st Int. workshop on movement pattern analysis (MPA) in conj. with the 6th Int. conf. on Geographic Information Science.

Versichele, M., Neutens, T., Claeys Bouuaert, M., Van de Weghe, N., 2014b. Time-geographic derivation of feasible co-presence opportunities from network-constrained episodic movement data. Transactions in GIS 18 (5), 687-703.

Versichele, M., Neutens, T., Delafontaine, M., Van de Weghe, N., 2012a. The use of bluetooth for analysing spatiotemporal dynamics of human movement at mass events: A case study of the ghent festivities. Applied Geography 32 (2), 208-220.

Versichele, M., Neutens, T., Goudeseune, S., Van Bossche, F., Van de Weghe, N., 2012b. Mobile mapping of sporting event spectators using bluetooth sensors: tour of flanders 2011. Sensors 12 (10), 14196-14213.

Vukovic, M., Lovrek, I., Kraljevic, H., 2012. Discovering shoppers' journey in retail environment by using RFID. In: KES. pp. 857-866.

Yamin, M., Ades, Y., 2009. Crowd management with rfid and wireless technologies. In: Networks and Communications, 2009. NETCOM'09. First International Conference on. IEEE, pp. 439-442. 
Zhou, S., Pollard, J. K., 2006. Position measurement using bluetooth. Consumer Electronics, IEEE Transactions on 52 (2), 555-558. 